\title{
Analysis of Use and Outcomes of Peripherally Inserted Central Catheter (PICC-Line) in Hemato-Oncological Patients
}

\author{
Sulav Sapkota*, Radheshyam Naik \\ Department of Medical Oncology, HCG PVT LTD, Bangalore, India \\ Email:*dr.sulavsapkota@yahoo.com
}

How to cite this paper: Sapkota, S. and Naik, R. (2018) Analysis of Use and Outcomes of Peripherally Inserted Central Catheter (PICC-Line) in Hemato-Oncological Patients. Journal of Cancer Therapy, 9, 35-41.

https://doi.org/10.4236/jct.2018.91005

Received: November 29, 2017

Accepted: January 22, 2018

Published: January 25, 2018

Copyright ( 92018 by authors and Scientific Research Publishing Inc. This work is licensed under the Creative Commons Attribution International License (CC BY 4.0).

http://creativecommons.org/licenses/by/4.0/

\begin{abstract}
Aims: To audit the use and outcomes of using PICC lines in hemato-oncological patients. Objectives: To study the demographics of patients: studying the use of PICC line in hemato-oncological patients; studying the rate of complications in PICC line; studying the cause of early removal of PICC line. Methods: All PICCs inserted in adult hemato-oncological patients in Hematology and Medical Oncology Department of Health Care Global (HCG) Hospital were studied prospectively, as per the proforma, till PICCs were removed or patient expired and the pattern of complications were noted. Results: Eighty-four PICCs were inserted over a period of initial nine months and followed for a total of 1 year with three months post insertion duration for a total of 10,868 catheter-days (mean of 129 days i.e. 4.3 months, range: 1 to 288 days). The most common indication for PICC was chemotherapy (100\%). Among them 19 (22\%) PICCs had complications and 12 were removed at the rate of 1.1/1000 PICC-days. Complications with haematologic malignancies were more as compared to those with solid tissue malignancies. Conclusions: Despite significant complication rates, PICCs are a relatively safe and cost effective mode of establishing central venous access.
\end{abstract}

\section{Keywords}

Peripherally Inserted Central Catheter (PICC), Vascular Access Device (VAD), Catheter-Related Bloodstream Infection (CR-BSI), Eastern Cooperative Oncology Group Performance Status (ECOG PS), Central Venous Catheter (CVC)

\section{Introduction}

The management of the patient with cancer demands stable venous access that is 
used for a wide range of indications. When the patient is felt to have poor venous access or when some chemotherapy drugs used in the treatment of cancer are not suitable to be administered into small veins in the hand or arm and these drugs must be administered in a larger vein for adequate dilution, peripherally inserted central catheter (PICC) line is best suited. PICC can be used for administration of the chemotherapy drugs by continuous infusion, which does not require a lengthy hospital stay and can be administered at home or when extended chemotherapy treatment is required. Other uses of PICC can be when there is anticipation of frequent needle sticks to obtain blood samples. It also eases patient's anxiety regarding frequent needle sticks.

Peripherally inserted central catheter (PICC) is a form of vascular access device (VAD) that can be used for a prolonged period of time for all kinds of infusate-chemotherapy, blood products transfusion, fluid and electrolytes infusion, total parenteral nutrition, antibiotics administration, stem cell infusion as well as access to the bloodstream for clinical monitoring and microbial culturing.

PICC is a catheter that enters the body through the skin at a peripheral site, extends to the superior vena cava, and stays in place for days or weeks. First described in 1975 [1] it is an alternative to central venous catheters such as subclavian catheters, internal jugular or femoral catheter, which have higher rates of infection and may result in pneumothorax and haemothorax while PICCs have no such issue because of the method of placement.

In hemato-oncological patients, use of PICC has provided a relatively simple, safe, easy, rapid and maintenance free means of accessing a central vein for the purposes of chemotherapy or other intravenous fluids irrespective of $\mathrm{pH}$, osmolarity, or other chemical properties of the solution or medication.

Few prospective studies evaluating the safety of PICCs have been performed despite their widespread use, and even fewer have focused on patients with cancer.

Therefore, we conducted this prospective study to determine the complication rate and safety of PICCs in cancer patients attending our hospital.

\section{Patients and Methods}

All the cancer inpatients were randomly selected at HCG hospital, who were deemed to have difficult venous access or required a central venous access for multitude of indications, particularly chemotherapy after recommendation by primary care physician were referred to our PICC Centre for PICC insertion. Insertion of $4 \mathrm{Fr}$ single lumen triple-valved PICC was done under strict aseptic conditions, under USG guidance. The PICC was inserted in any of the major vein of the upper extremities, more frequently in basilic vein and secured by stat-lock adhesive dressing and sutures.

We prospectively studied and followed all patients who had a PICC successfully inserted during 1 year period, from June 15, 2015, to June 14, 2016 with initial 9 months for insertion and later 3 months for follow-up, until they were removed or patient had expired. The study was approved by the Central Ethics Committee. Categorical variables were analyzed for their frequency distribution. 


\subsection{Inclusion Criteria}

Age: 14 - 65 yrs

Treatment naive and those undergoing Treatment.

ECOG PS $<2$

Histologically confirmed malignancy

EXCLUSION CRITERIA:

Coagulopathy

Thrombosis

\subsection{Statistical Analysis}

The sample size was calculated on the basis of sample size formula for the qualitative study.

All the parameters related to PICC line and patients i.e. name, age, sex, date of insertion and removal of PICC, diagnosis, patient status, time taken for insertion, length and depth of PICC line, number of attempts for insertion, type of vein accessed, complication (if any), PICC dwell days, were studied and analysed. Categorical variables were analyzed for their frequency distribution. For the analysis, each PICC placement was counted as a new event. Therefore, all presented calculations, including the demographic description of the patient group, used PICC placements rather than individual patients as the unit for counting.

In case of Lost to follow up patients, last visit was taken as the date of removal of PICC line.

\section{Observation \& Result}

A total of eighty one patients were considered for PICC line insertion during the study period, of which two insertions failed due to inability to pass the guide wire. Out of the remaining seventy nine patients, five patients had PICC line inserted twice and two patients were lost to follow up after insertion during observation period. All the patients were analysed and evaluated for the study. The demographic profile of 79 patients and the primary indications for PICC placement are listed in Table 1.

Overall, the patient population included 38 (48\%) men and 41 (52\%) women, with a mean age of 45 years (range: 14 to 65 years). 43 patients (55\%) had solid, while $36(45 \%)$ had haematological malignancies. Among the solid tumours, the most common were GIT malignancies, (38\%). Acute myeloid leukaemia and Non Hodgkin Lymphoma were the most common (34\% each) among the haematological malignancy group. The basilic vein was used most frequently, in 58 patients (70\%) for the placement of PICC. 79 patients (100\%) were discharged from Hospital with a PICC in situ. All the patients were followed up on weekly interval with PICC team.

About 70 patients $(88 \%)$ were treated on OPD basis in-between the chemotherapy period and rest $9(12 \%)$ patients were hospitalised for the entire duration of PICC use. 
Table 1. Characteristics of the patients receiving a PICC $(n=79)$.

\begin{tabular}{ccc}
\hline characteristic & No. of patients & $\%$ \\
\hline Age (years) & 8 & \\
$14-24$ & 13 & 10 \\
$25-34$ & 14 & 18 \\
$35-44$ & 13 & 17 \\
$45-54$ & 31 & 39 \\
$55-65$ & &
\end{tabular}

Mean age: 45 years

\begin{tabular}{|c|c|c|}
\hline Sex & & \\
\hline Male & 38 & 48 \\
\hline Female & 41 & 52 \\
\hline \multicolumn{3}{|l|}{ Underlying cancer } \\
\hline Solid tumor & 43 & 55 \\
\hline Breast cancer & 12 & 28 \\
\hline GIT \& PB tract & 16 & 38 \\
\hline Head \& Neck & 9 & 21 \\
\hline Gynae cancer & 3 & 7 \\
\hline Lung cancer & 2 & 4 \\
\hline Others & 1 & 2 \\
\hline Haematological malignancies & 36 & 45 \\
\hline AML & 12 & 34 \\
\hline NHL & 12 & 34 \\
\hline ALL & 6 & 16 \\
\hline Multiple myeloma & 3 & 8 \\
\hline Others & 3 & 8 \\
\hline \multicolumn{3}{|l|}{ Site of PICC } \\
\hline Right basilic vein & 10 & 12 \\
\hline Left basilic vein & 48 & 58 \\
\hline Right cephalic vein & 1 & 1 \\
\hline Left cephalic vein & 12 & 14 \\
\hline Right brachial vein & 11 & 13 \\
\hline Left brachial vein & 2 & 2 \\
\hline Complications & 19 & 22 \\
\hline CR BSI & 2 & 2 \\
\hline Phlebitis & 5 & 6 \\
\hline Blockage & 3 & 4 \\
\hline Accidental removal & 2 & 2 \\
\hline
\end{tabular}


Continued

\begin{tabular}{ccc}
\hline Leakage & 2 & 2 \\
Thrombosis & 3 & 4 \\
Abnormal position & 1 & 1 \\
Hematoma & 1 & 1 \\
No complications & 66 & 78 \\
Total PICCs removed & 38 & 46 \\
Removal due to death & 15 & 19 \\
$\begin{array}{c}\text { Removal due to complications } \\
\text { Removal after completion of } \\
\text { treatment without } \\
\text { complications }\end{array}$ & 12 & 14 \\
\hline
\end{tabular}

The 84 PICCs were in place for a total of 10,868 catheter-days (mean of 129 days, i.e. 4.3 months and range, 1 to 288 days). The most common indication for PICC was chemotherapy (100\%). The most frequently used chemotherapy regimen was adjuvant chemotherapy for Gastrointestinal cancer in solid cancers and induction chemotherapy for acute myeloid leukaemia in haematological cancers. Majority of patients preferred PICC in this section.

Demographic and disease characteristics of the 79 patients receiving a PICC and the reason for PICC removal are given in Table 1. Total 38 PICCs were removed, out of which 15 (19\%) were removed due to death of the patients due to progression of disease. PICCs in 12 (14\%) patients were removed due to complications. 11 (13\%) PICCs were removed at completion of therapy without any evidence of complication.

PICCs of patients with Hematological malignancies 11 (13\%) were found to have more complications than solid malignancies 8 (9\%) in our study.

Total 19 (22\%) complications occurred during our study at the rate of 1.74 per 1000 catheter-days, of which Phlebitis was the most common complication. Blockage was more common in solid malignancies while thrombosis was most common in haematological malignancies. Only 2 patients had infection and both were haematological cases. During the study, $2 \%$ cases of PICC had positive blood culture which showed growth of Staphylococcus and Pseudomonas. Antibiotics used commonly were Vancomycin and Carbepenems for catheter-related bloodstream infection (CR-BSI) while oral Clarithromycin for phlebitis treatment.

\section{Discussion}

PICCs are now becoming more popular than other Central Venous Catheters (CVCs), because of ease of insertion, removal, cost-effectiveness and low procedure related complications. PICCs have made administration of chemotherapy much safe and reliable. However its complications in hemato-oncological patients have not been much studied, therefore we studied prospectively its com- 
plication rate in cancer patients.

In our study the subjects were taken between age groups 14 to 65 years. Maximum numbers of patients in the current study fall in the age group of 55 65 years (overall mean 45 years) Table 1 . This is in contrast to the study of Ghulam Haider et al. [2] (2009) where the maximum number of patients were in the age group of 14 - 40 years. This discrepancy could be because haematological malignancies like AML, NHL and solid malignancies like GIT, Breast Cancers were more common in our study which are found to be in this age group.

In our study, complication rate was found to be $22 \%$ (Table 1) which is lesser to that found by Walsh L.J. et al. [3] that was $32.8 \%$. This finding could be because of the difference in the sample sizes of the two studies. However the pattern of complication was different in the two studies.

In the present study the complication rate was seen more in haematological group while a retrospective study conducted by K. Cheong et al. [4] showed more complications in solid tumour group, this discrepancy could be because of prolong hospitalisation, long duration of chemotherapy and use of steroid in haematological malignancies than solid tumour.

In a prospective study of 251 ICU patients conducted by Safdar et al. [5], the author found a complication rate similar to other Subcutaneously implanted CVCs. PICC related infection rate was $2.4 \%$, a rate of $2.1 / 1000$ catheter-days.

The complication rate seems to depend on the infusate being given through the PICC. When PICCs were used only for IV antibiotics, the complication rate were found to be low as compared to those where multiple infusates were being given through the PICC for example chemotherapy, blood products and electrolyte infusion and antibiotics, resulting in the higher rates of complications.

In the present study, complication rate was found to be more common in Haematological malignancies than in solid malignancies. This observation is consistent with the findings of Walshe L.J. et al. (2002) [3]. Thrombotic complication in the present study was less as compared to Walshe L.J. et al. [3], Cheong et al. [4] and Chu et al. [6]. This discrepancy in the result could be because in the present study before every catheter insertion, bleeding and coagulation profile was routinely checked and only those with normal study were offered insertion. Strict aseptic measure was taken while inserting the catheter, with least possible number of attempts. Prompt PICC care, regular flushing with normal saline was done on weekly basis and especially after every antibiotics and blood products infusions.

\section{Conclusion}

PICC provides very safe, easy and cost-effective central venous access in cancer patients. Peripherally inserted central catheter complication rates can be minimized if inserted under aseptic condition and maintained on regular basis. Both, patients and treating team, should take the responsibility of the PICC line. This also includes patient's family and staff education with formal aftercare guide- 
lines, strict infection control and regular PICC maintenance and surveillance, making them safe and acceptable. However a prospective study is needed to compare the quality of life, financial issues and social issues of patients with PICC.

\section{Conflict of Interest}

This article has no potential conflict of interest.

\section{Ethical Approval}

All procedures performed in studies involving human participants were in accordance with the ethical standards of the institutional and/or national research committee.

\section{Informed Consent}

Informed consent was obtained from all individual participants included in the study.

\section{References}

[1] Hoshal, V.L. (1975) Total Intravenous Nutrition with Peripherally Inserted Silicone Elastomer Central Venous Catheters. Archives of Surgery, 110, 644-646. https://doi.org/10.1001/archsurg.1975.01360110190032

[2] Haider, G. (2009) Determination of Complication Rate of PICC Lines in Oncological Patients. Journal of Pakistan Medical Association, 59, 663-667.

[3] Walshe, L.J., Malak, S.F., Eagon, J. and Sepkowitz, K.A. (2002) Complication Rates among Cancer Patients with Peripherally Inserted Central Catheters. Clinical Oncology, 20, 3276-3281. https://doi.org/10.1200/JCO.2002.11.135

[4] Cheong, K., Perry, D., Karapetis, C. and Koczwara, B. (2004) High Rates of Complications Associated With Peripherally Inserted Central Catheters in Patients with Solid Tumours. Internal Medicine Journal, 34, 234-238. https://doi.org/10.1111/j.1444-0903.2004.00447.x

[5] Safdar, N. and Maki, D.G. (2005) Risk of Catheter Related Blood Stream Infection with Peripherally Inserted Central Venous Catheters Used in Hospitalized Patients. Chest, 128, 489-495. https://doi.org/10.1378/chest.128.2.489

[6] Chu, F.S., Cheng, V.C., Law, M.W. and Tso, W.K. (2007) Efficacy and Complications in Peripherally Inserted Central Catheter Insertion: A Study Using 4-Fr Non-Valved Catheters and a Single Infusate. Australasian Radiology, 51, 453-457. https://doi.org/10.1111/j.1440-1673.2007.01870.x 